Meta

Journal des traducteurs

Translators' Journal

\title{
Comment formuler une définition terminologique
}

\section{Louise Larivière}

Volume 41, numéro 3, septembre 1996

URI : https://id.erudit.org/iderudit/003401ar

DOI : https://doi.org/10.7202/003401ar

Aller au sommaire du numéro

\section{Éditeur(s)}

Les Presses de l'Université de Montréal

ISSN

0026-0452 (imprimé)

1492-1421 (numérique)

Découvrir la revue

Citer cet article

Larivière, L. (1996). Comment formuler une définition terminologique. Meta, 41(3), 405-418. https://doi.org/10.7202/003401ar

\section{Résumé de l'article}

Cet article précise la "vraie" nature d'une définition terminologique. Dans un premier temps, on montre que les ouvrages de référence sur la langue ne peuvent être classifies par le type de définition qu'ils utilisent ni du point de vue de la composition, ni de celui de la structure mais bien du point de vue de sa finalité. On étudie ensuite la nature spécifique de la définition terminologique en tant que structure relationnelle des concepts; puis on présente une façon de formuler ce type de définition à l'aide d'éléments d'inclusion et de distinction pour ensuite définir le degré d'exhaustivité qu'elle doit posséder pour pouvoir faire comprendre un concept donné en plus de permettre l'utilisation de ce concept dans un but spécifique. Enfin, on décrit le thésaurus terminologique comme le produit de référence sur la langue le plus approprié pour l'indexation des termes en terminologie et en documentation. Les exemples sont tirés de documents professionnels,
Ce document est protégé par la loi sur le droit d'auteur. L'utilisation des services d'Érudit (y compris la reproduction) est assujettie à sa politique d'utilisation que vous pouvez consulter en ligne.

https://apropos.erudit.org/fr/usagers/politique-dutilisation/ 


\title{
COMMENT FORMULER UNE DÉFINITION TERMINOLOGIQUE
}

LOUISE LARIVIÈRE

Université de Montréal et Université Concordia, Montréal, Canada

\begin{abstract}
Résumé
Cet article précise la «vraie» nature d'une définition terminologique. Dans un premier temps, on montre que les ouvrages de référence sur la langue ne peuvent être classifiés par le type de définition qu'ils utilisent ni du point de vue de la composition, ni de celui de la structure mais bien du point de vue de sa finalité. On étudie ensuite la nature spécifique de la définition terminologique en tant que structure relationnelle des concepts; puis on présente une façon de formuler ce type de définition à l'aide d'éléments d'inclusion et de distinction pour ensuite définir le degré d'exhaustivité qu'elle doit posséder pour pouvoir faire comprendre un concept donné en plus de permettre l'utilisation de ce concept dans un but spécifique. Enfin, on décrit le thésaurus terminologique comme le produit de référence sur la langue le plus approprié pour l'indexation des termes en terminologie et en documentation. Les exemples sont tirés de documents professionnels.
\end{abstract}

\begin{abstract}
This paper specifies the "true" nature of a terminological definition. First, it shows that language reference works cannot be identified by the definition they are using considered from the point of view of its composition, nor from the point of view of its structure, but from the point of view of its finality. Then, it presents the specific nature of the terminological definition as the extension of the relational structure of concepts; it shows how to formulate this type of definition by the mean of inclusion and distinctive features; and defines the degree of exhaustivity it should possess in order to be able to establish the comprehension of a concept as well as to use this concept for a specific purpose. It finally describes the terminological thesaurus as the most appropriate language reference work to index terms for terminology as well as documentation purposes. Examples used are taken from the field of professional documents.
\end{abstract}

Key words : terminological definition, terminological thesaurus, relational structure of concepts, professional documents

\section{INTRODUCTION}

La définition terminologique (DT) existe-t-elle vraiment? Dans les années 70 , certains nient son existence en jugeant «artificielle» 1'opposition entre terminologie et lexicographie (Dubois 1979: 57) et en envisageant même la terminologie non pas comme une discipline distincte mais comme une lexicographie technique (Guilbert cité par Dubois 1979: 57). Pour d'autres, sa place est «loin d'être nette»: la DT est une sorte de «compromis entre définition lexicographique et description encyclopédique» dont la pratique en terminographie «rejoint celle d'une lexicographie spéciale» (Rey 1979: 42-44).

Dans les années 80 , Rousseau (1983: 35) constate que l'on a peu écrit sur la DT et se demande «si ce n'est pas à cause de la difficulté d'en donner une description générale suffisamment cohérente et spécifique». Il se demande également s'il ne serait pas préférable et plus prudent de parler de «définition en terminologie» plutôt que de «défini- 
tion terminologique». De Bessé (1990:252), quant à lui, s'interroge sur l'existence d'une DT et, en admettant qu'elle existe, cherche à savoir quelles sont ses caractéristiques, ses ressemblances et ses différences avec la définition lexicographique (DL) et la définition encyclopédique (DE) et quels sont ses principes de rédaction. Il trouve difficile de répondre à ces questions étant donné le peu de documentation existant sur le sujet. Toutefois, note de Bessé, si l'on a peu écrit sur la DT, on l'a beaucoup utilisée comme l'attestent de nombreuses définitions qualifiées de «terminologiques» dans plusieurs répertoires et banques de données.

Les années 90 seront-elles celles de la DT? En posant au départ que la DT existe, nous allons montrer comment elle pourrait se présenter et tenter, accessoirement, de démontrer:

1) que la DT se distingue de la DL et de la DE, non pas tant par sa structure ni par sa composition, mais par sa finalité;

2) que la DT constitue un prolongement de la structure relationnelle des concepts et que, de ce fait, la formulation de la définition sera, à la fois, fonction du type de relations qui existent entre les concepts et fonction de la nature même du concept, et ce, quelle que soit la place que ce dernier occupe à l'intérieur d'un système hiérarchisé.

Les exemples utilisés aux fins de cet exposé sont tirés de la terminologie de documents professionnels.

\section{RÉPERTOIRES ET COMPOSITION DES DÉFINITIONS}

Est-ce qu'un répertoire linguistique se reconnaît à un type de définition envisagée du point de vue de sa composition? Avant de répondre à cette question, il convient d'abord de préciser quelles sont ces définitions que nous envisageons du point de vue de leur composition. On en distingue généralement trois :

a. la définition lexicale que certains appellent «nominale» et qui se compose d'une périphrase synonymique :

ex. : Aérogramme = lettre acheminée par avion;

b. la définition conceptuelle qui se compose d'un énoncé comprenant un terme de base ou incluant (genre prochain, tout, classe ou catégorie) et des propositions explicitant les traits sémantiques (différenciateurs et essentiels) d'une notion:

ex. : Attache (de) signature = ensemble d'éléments qui suit la formule de politesse, avec laquelle elle forme la souscription d'une lettre, et qui sert à identifier la personne expéditrice;

c. la définition référentielle qui se compose de l'une ou l'autre des définitions, lexicale et conceptuelle, suivie d'un développement sous forme d'une description comportant les traits accessoires de la notion:

ex.: En-tête (de) lettre = inscription imprimée de la raison sociale ou de la dénomination officielle d'un organisme expéditeur. Elle a une double fonction: fournir aux destinataires l'identité de l'organisme expéditeur (rôle utilitaire) et une bonne image de marque de ce dernier (rôle publicitaire), se place généralement au haut du papier à lettres et peut comprendre les éléments principaux suivants présentés les uns sous les autres ou en ligne continue: nom, adresse postale et télégraphique, numéros de téléphone, de télécopieur et de télex.

Elle peut aussi comprendre le slogan de l'entreprise ou des mentions publicitaires, son logo ou tout autre graphisme, ses niveaux hiérarchiques allant du général au particulier, son statut juridique, l'adresse du siège social, des bureaux régionaux et des succursales, le 
nom des membres du conseil d'administration, l'énumération de ses produits et l'indication de ses heures d'ouverture.

Seule la première page de la lettre se présente sur du papier à en-tête.

Examinons maintenant les définitions de «contrat» dans les entrées de quatre types de répertoires linguistiques: 1) un vocabulaire spécialisé, Le vocabulaire de la fonction commerciale par Hydro-Québec (v. annexe I); 2) un petit dictionnaire de langue, le Petit Larousse illustré (v. annexe II); 3) un grand dictionnaire de langue, le Robert électronique (v. annexe III); et 4) un dictionnaire encyclopédique, le Grand dictionnaire encyclopédique Larousse (v. annexe IV). Examinons du même coup la composition de chacune de ces entrées.

On trouve des définitions conceptuelles dans le vocabulaire spécialisé, ainsi que des définitions conceptuelles en plus des définitions lexicales et même implicites dans les dictionnaires de langues, petits et gros, et dans le dictionnaire encyclopédique. Quant aux encyclopédies dont on n'a pas encore parlé, elles comportent des entrées qui sont davantage des descriptions de choses ou, si l'on veut, des définitions élargies (extended definitions) ou encore des définitions référentielles, dont la structure textuelle est un discours suivi s'opposant à l'éclatement des messages (Lehmann 1990: 218).

Quant à la composition des entrées, elle varie plus ou moins selon la nature des répertoires. Toutes les entrées comprennent l'unité d'entrée et une ou des définitions. Les dictionnaires donnent le sens de chacune des acceptions des termes, les emplois particuliers avec leur définition respective et, selon le dictionnaire, les traits phonétiques, les traits grammaticaux de genre et de nombre, l'étymologie, des collocations, d'autres termes du même champ sémantique, des renvois à des citations à l'aide de numéros, les termes composés ou dérivés. Le vocabulaire comprend un renvoi «voir aussi» et le dictionnaire encyclopédique une section dite encyclopédique qui décrit, en détail, certaines notions à l'intérieur de domaines particuliers.

Les répertoires ne se caractérisent donc pas, nécessairement, par une sorte de définition bien que, en général, la définition conceptuelle se retrouve dans les vocabulaires, que la définition lexicale soit le propre des dictionnaires de langue et que la définition référentielle, le propre des dictionnaires encyclopédiques et des encyclopédies ${ }^{1}$. C'est davantage par la composition de leurs entrées que par celle de leurs définitions que se distinguent ces divers répertoires.

\section{RÉPERTOIRES ET STRUCTURE DES DÉFINITIONS}

Les répertoires se distinguent-ils, par ailleurs, par la structure de leurs définitions? Posons d'abord qu'une définition structurée est une définition qui possède un terme incluant lui permettant de rattacher une notion à une autre. Les définitions se distinguent d'après leur structure parce qu'il existe des relations entre les unités et que ces relations déterminent la structure de la définition qui en est le prolongement. À la suite de Dahlberg (1983: 19-20 et 25-26), nous distinguerons les définitions suivantes :

a. la définition générique qui s'utilise pour marquer le rapport de genre à espèce entre deux notions:

ex. : Document $($ de $)$ correspondance $=$ document professionnel qui sert à échanger des messages d'affaires de caractère professionnel ou privé.

où l'incluant «document professionnel» est le genre prochain;

b. la définition partitive qui s'utilise pour marquer le rapport de tout à partie entre deux notions : 
ex. : Compléments (de la) lettre = ensemble des éléments d'identification et de destination qui suivent le corps de la lettre.

où l'incluant «ensemble des éléments» est le tout;

c. la définition catégorielle qui s'utilise pour marquer le rapport d'une notion avec une catégorie de pensée ou une classe d'objets :

ex. : Courrier électronique = procédé de transmission à distance, sans support physique, de documents alphanumériques ou graphiques au moyen de systèmes électroniques reliés par des lignes téléphoniques.

où l'incluant «procédé» est une catégorie ou un genre suprême;

ex. : Document professionnel $=$ tout document utilisé par des institutions ou des individus à des fins de gestion courante, de recherche ou de développement.

où l'incluant «document» est une classe.

Voyons des exemples de ces définitions dans divers répertoires linguistiques.

\section{Vocabulaires}

ex. Connaissement $=$ document remis par un transporteur à un destinataire attestant la prise en charge des marchandises et leur livraison par ce transporteur (Globensky 1980: 79).

Définition générique ou catégorielle où «document» est le genre prochain ou une classe selon le plan de classement utilisé.

ex. Devis (estimatif) $=$ document comportant une désignation succincte de la nature de la prestation d'un marché, la quantité prévue, le prix unitaire et la somme représentée (de Villers $1981: 30$ ).

Définition générique ou catégorielle où «document» est le genre prochain ou une classe selon le plan de classement utilisé.

ex. Norme $=$ formule qui définit un type d'objet, un produit, un procédé technique en vue de simplifier, de rendre plus efficace et plus rationnelle la production. (de Villers $1981: 60$ ).

Définition catégorielle où «formule» est une catégorie.

\section{Grand Dictionnaire Encyclopédique Larousse}

ex. Appel (d') offres = mode de passation des marchés publics dans lequel l'Administration conserve le choix de son cocontractant après avoir mis publiquement en concurrence les candidats.

Définition catégorielle où «mode» est une catégorie.

ex. Formule $=$ imprimé de modèle standard qui sert à certaines formalités administratives.

Définition générique où «imprimé» est le genre prochain.

ex. Norme $=$ règle [de conduite] fixant les conditions de la réalisation d'une opération, de l'exécution d'un objet ou de l'élaboration d'un produit dont on veut unifier l'emploi ou assurer l'interchangeabilité.

Définition générique où «règle de conduite» est le genre prochain ou la définition catégorielle où «règle» est une catégorie. 


\section{Le Robert électronique}

ex. Devis = état détaillé des travaux à exécuter avec estimation des prix.

Définition catégorielle où «état» est une catégorie.

ex. Formule = imprimé type destiné à être rempli par les usagers pour certaines formalités administratives.

Définition générique où «imprimé» est le genre prochain.

ex. Norme française $=$ document de référence qui définit l'ensemble des prescriptions techniques concernant des produits ou des méthodes déterminés.

Définition générique où «document de référence» est le genre prochain.

Les définitions génériques et catégorielles se retrouvent dans tous les types de répertoires et les définiitons partitives le pourraient également. Ces définitions ne sont donc pas fonction d'un type de répertoire et ne caractérisent pas nécessairement ces derniers.

\section{RÉPERTOIRES ET FINALITÉ DES DÉFINITIONS}

Si les répertoires ne se reconnaissent ni à la composition ni à la structure des définitions, ils se reconnaissent, toutefois, à la finalité de ces dernières. Il existe trois types de définitions quant à la finalité :

a. la définition lexicographique (DL), utilisée dans les dictionnaires de langue et les dictionnaires encyclopédiques, qui se propose d'expliciter des signifiés en distinguant les sens et les emplois des signes (ou mots) d'une langue;

b. la définition encyclopédique (DE), utilisée dans les encyclopédies et les dictionnaires encyclopédiques, qui se propose de fournir un ensemble de connaissances sur une chose;

c. la définition terminologique (DT), utilisée dans les vocabulaires spécialisés, qui se propose de caractériser (i.e. de délimiter et de distinguer des autres notions) des notions dénommées par un terme et représentant une chose à l'intérieur d'un système organisé.

Ainsi, ces définitions se distinguent par leur finalité respective: expliciter des signifiés (DL), fournir des connaissances (DE), caractériser des notions (DT). Elles se distinguent, du même coup, par leur objet : le signe ou mot (DL), la chose (DE), la notion (DT), ainsi que par leur produit: dictionnaire de langue et dictionnaire encyclopédique (DL), dictionnaire encyclopédique et encyclopédie (DE), vocabulaire spécialisé (DT). Leurs différences tiennent donc à leur nature et non à leur formulation liée soit à la composition soit à la structure. Elles se distinguent également par la forme de leurs entrées.

\section{SPÉCIFICITÉ DE LA DÉFINITION TERMINOLOGIQUE}

Pour bien circonscrire la nature de la définition terminologique et voir, plus spécifiquement, ce qui la distingue des autres définitions, il faut d'abord établir le but de la terminologie. Pour Rousseau (1983: 38), «la raison d'être de la terminologie est avant tout de savoir nommer les choses dans une perspective de connaissance (aspect cognitif) et d'expression ou de communication».

Le concept ou notion se trouve donc à la base du processus terminologique. Il se définit comme une représentation mentale d'un référent (ou chose) dénommée par un terme et explicitée, à la fois, par la place qu'il occupe au sein d'un système organisé de relations et par une définition. On peut schématiser le concept comme suit: 


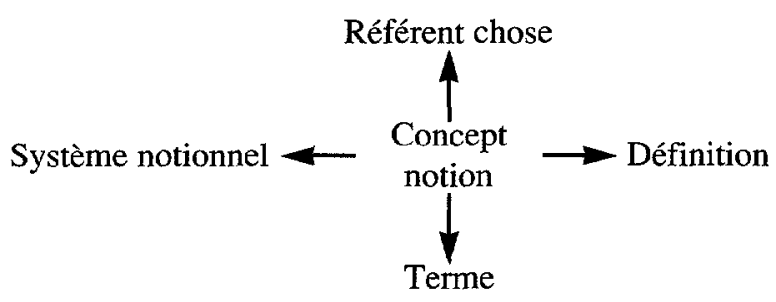

Figure 1: Schéma du concept (notion)

Ainsi, pour un domaine donné, chaque notion occupe une place définie à l'intérieur d'un système organisé de relations. C'est cette place qui, au premier chef, permet de caractériser la notion qui va, par suite, s'expliciter par sa définition. Il se doit donc d'y avoir un lien entre les deux ${ }^{2}$. Par conséquent, la formulation des définitions sera, nécessairement, fonction de la place qu'occupent les notions au sein de leur structure relationnelle et en constituera le prolongement, comme l'a démontré McNaught (1982: 54). Ce lien entre système notionnel et définition est tel que si on change la place d'une notion dans le système, on devra, par suite, changer sa définition.

Nous croyons que le système organisé de relations devrait être hiérarchique de préférence à thématique. Cette question fera l'objet d'une communication subséquente. Disons, pour le moment, que la structure hiérarchique rend la formulation de la définition :

(1) plus facile parce qu'elle est motivée: on n'a qu'à utiliser, comme point de départ de la définition, l'incluant approprié (genre, tout, catégorie ou classe) et à poursuivre à l'aide des traits différenciateurs de la notion à définir;

(2) plus cohérente: on ne risque pas d'avoir une même notion reprise à un autre endroit (polyhiérarchie) et d'aboutir à un polymorphisme définitionnel qui découle du fait que l'on ne définisse pas les notions à l'intérieur d'un système d'inclusion.

En fait, il n'y a que la DT qui puisse véritablement s'utiliser à l'intérieur d'un système fini et organisé de notions dont nous reproduisons un exemple à la figure 2 .

\section{FORMULATION DE LA DÉFINITION TERMINOLOGIQUE}

Comme la DT se définit en fonction de sa finalité et non de sa forme, elle adoptera plusieurs formulations quant à sa structure (définition générique, partitive ou catégorielle) et quant à sa composition (définition lexicale, conceptuelle ou référentielle). Ces formulations n'ont rien à voir avec la place qu'occupe la notion à l'intérieur du système, bien que plus une notion se situe au bas de l'échelon d'une hiérarchie, plus sa définition aura tendance à être une paraphrase du terme au lieu d'en contenir les traits différenciateurs.

Voyons la définition de quelques notions (v. figure 3) qui apparaissent dans la liste systématique hiérarchisée présentée plus haut. Quelle que soit la formulation de ces définitions, ces dernières n'en demeurent pas moins des DT parce qu'elles caractérisent des notions dénommées par un terme, de façon univoque, à l'intérieur d'un système hiérarchisé de notions. 


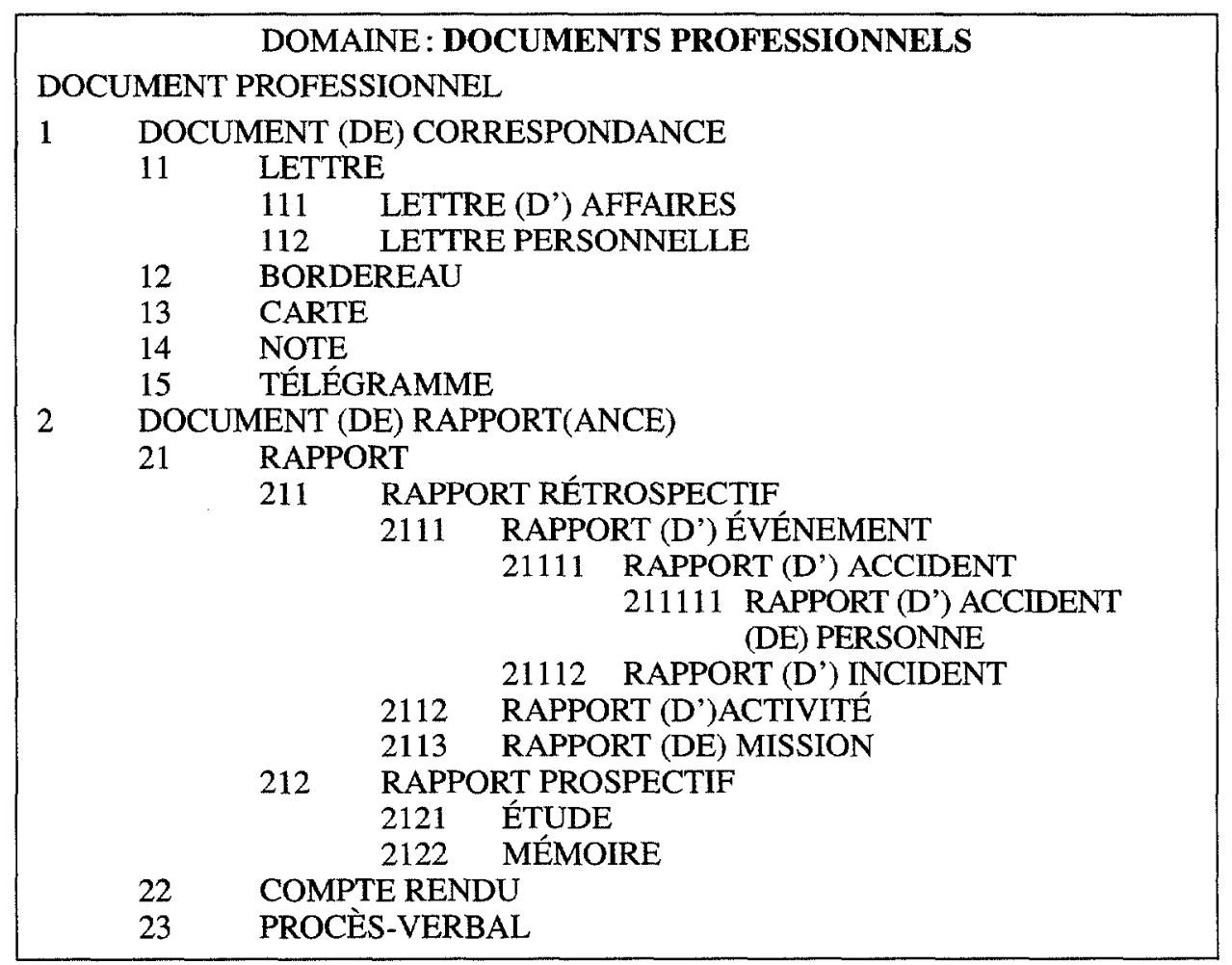

Figure 2: Liste systématique hiérarchisée de notions (partielle) 
DOCUMENT PROFESSIONNEL = Tout document utilisé par des institutions ou des individus à des fins de gestion courante, de recherche ou de développement.

DOCUMENT (DE) CORRESPONDANCE = Document professionnel qui sert à échanger des messages d'affaires de caractère professionnel ou privé.

LETTRE = Document de correspondance externe, de caractère personnel, écrit à la main ou imprimé, généralement expédié sous enveloppe (parfois à découvert) à un ou plusieurs destinataires (institutions ou individus, identifiés ou non) et soumis à un protocole de présentation strict dit protocole épistolaire. La lettre se compose d'éléments essentiels et accessoires regroupés en trois sections: les préliminaires, le corps et les compléments.

LETTRE (D') AFFAIRES = Lettre qui traite des activités et des occupations professionnelles d'institutions et d'individus, qui est rédigée en fonction de buts et de destinataires déterminés et servant à des fins d'échanges divers selon un formalisme, un style et un vocabulaire propres.

DOCUMENT (DE) RAPPORT(ANCE) = Document professionnel qui sert à relater une activité.

RAPPORT $=$ Document de rapport, à caractère incitatif, de taille variable, portant sur une question particulière et qui vise à fournir des données à une autorité responsable appelée à prendre une décision et, le cas échéant, les mesures nécessaires.

RAPPORT RÉTROSPECTIF = Rapport relatant une réalisation passée.

RAPPORT (D') ÉVÉNEMENT = Rapport rétrospectif qui évalue un fait fortuit important.

RAPPORT (D') ACCIDENT = Rapport d'événement qui expose les circonstances qui entourent un accident, qui en recherche les causes et qui propose des mesures préventives.

\section{Figure 3: Définitions}

Par ailleurs, les traits sémantiques différenciateurs, utilisés dans la formulation d'une définition, s'établissent en fonction de traits catégoriels qui sont des relations qualitatives formelles (Dahlberg 1983: 25-26). Ces derniers permettent de faire une analyse systématique des notions et d'en arriver à rédiger les définitions de façon plus satisfaisante (Rousseau 1983: 43) en en facilitant la formulation. Ainsi, à chacune des catégories peut correspondre un certain vocabulaire (illustration non-exhaustive) :

entité : consiste en

composition : comprend, se compose de

localisation : s'inscrit, se place

forme: se présente

fonction : vise à, utilisé pour, sert de, sert à

propriété : formulations diverses

Voyons un exemple de définition avec son analyse en traits catégoriels (figure 4). 
VEDETTE = Mention obligatoire des préliminaires de la lettre qui comprend les coordonnées des destinataires: nom, titre, adresse et qui, dans son usage nordaméricain, s'inscrit contre la marge de gauche à quelques lignes au-dessous de la mention de date, alors que dans son usage européen, elle se place, à droite, en dessous de la date.

\section{TRAITS CATÉGORIELS} entité

propriété

composition

propriété

localisation

propriété

localisation

\section{ANALYSE}

\section{INCLUANT}

mention, préliminaires de la lettre

TRAITS SÉMANTIQUES

obligatoire

qui comprend les coordonnées des destinataires: nom, titre, adresse

dans son usage nord-américain

s'inscrit contre la marge de gauche à quelques lignes au-dessus de la mention date

dans son usage européen

se place, à droite, en dessous de la date

Figure 4 : Définition de «vedette» et analyse de cette définition

\section{EXHAUSTIVITÉ DE LA DÉFINITION TERMINOLOGIQUE}

Où commence et où s'arrête la pertinence dans l'ênumération des traits sémantiques? Pour y répondre, représentons-nous ce que veut savoir une personne lorsqu'elle demande: «Qu'est-ce qu'une 'vedette' dans une lettre ?». Cette personne veut savoir quelle est la réalité nommée par ce terme «vedette» faisant partie de la lettre ou, en d'autres termes, ce qu'elle doit apprendre sur cette réalité pour que, en en ayant une idée suffisamment juste, elle puisse l'utiliser correctement dans une lettre.

La réponse à cette personne sera de lui définir ce qu'est une «vedette» dans une lettre ou, en d'autres termes, de lui énumérer ce que sont les caractères de la notion «vedette» lorsqu'on l'utilise dans une lettre, et ce, de façon suffisante pour qu'elle puisse distinguer cette notion de toute autre notion et l'appliquer correctement lorsqu'elle rédige une lettre.

Ainsi, définir consistera à énumérer les caractères d'une notion pour pouvoir: 1) établir sa compréhension (i.e. la distinguer des autres notions); 2) utiliser cette notion à une fin donnée.

Quelles informations seront alors suffisantes pour atteindre ces deux objectifs: compréhension du concept et réutilisation? Selon de Bessé (1990:254), à la différence du développement encyclopédique, la définition terminologique s'arrête quand elle a donné toutes les informations permettant de situer et de différencier un concept à l'intérieur d'un système conceptuel. Pour le terme qui nous occupe, «vedette», la définition permettant de jouer ce rôle sera: Mention obligatoire des préliminaires de la lettre qui comprend les coordonnées des destinataires.

Cette définition est cependant insuffisante pour pouvoir utiliser cette notion dans un contexte donné. Il faut donc utiliser tous les traits sémantiques de la notion qui, dans ce cas-ci, ne sont pas accessoires mais essentiels à la deuxième finalité de la DT, soit: nom, titre, adresse et qui, dans son usage nord-américain, s'inscrit contre la marge de 
gauche à quelques lignes au-dessous de la mention de date, alors que dans son usage européen, elle se place, à droite, en dessous de la date.

Les traits utilisés ici sont plus que des «éléments encyclopédiques inutiles»; ils sont des éléments «importants» destinés à remplir «une fonction pédagogique» (Bessé 1990 : 254). Ce sont des traits essentiels à la double finalité de la DT33.

D'où, si l'on prend l'exemple de «contrat» (v. annexe I, II et III), il y a toute une gamme de possibilités entre la définition lexicale minimale: «convention entre des parties» et un cours de droit civil complet sur les obligations. Cette gamme existe pour répondre à des besoins variés.

\section{PRÉSENTATION DE LA DÉFINITION TERMINOLOGIQUE À L'INTÉRIEUR D'UN RÉPERTOIRE PARTICULIER: LE THÉSAURUS TERMINOLOGIQUE}

Sager (1982) a démontré les avantages de recourir à un type de répertoire qu'il nomme «thésaurus terminologique». McNaught (1982) et Larivière (1989) en ont donné des applications. Ce type de répertoire contient, dans chacune des parties qui le composent, les deux façons de caractériser une notion:

a. la structure relationnelle de toutes les notions qui se retrouve dans la liste systématique hiérarchisée;

b. la définition de toutes les notions qui se retrouve dans la liste alphabétique structurée et découlant de la structuration des notions.

Il fournit, de plus, une image globale d'une notion avec toutes ses relations et peut servir à d'autres fins que terminologiques: documentaires, pédagogiques, etc. La structure d'une entrée de thésaurus terminologique comporte les trois parties suivantes:

(1) Un énoncé définitoire (DE)

(2)Des relations

(a) Relations préférentielles

- Interlinguistiques : traductions (TR)

- Intralinguistiques : termes équivalents (TE)

(b)Relations hiérarchiques

- Hyperonymes (genre, catégorie, tout) : termes génériques (TG)

- Hyponymes (espèce, partie) : termes spécifiques (TS)

- Co-hyponymes: termes coordonnés (TC)

(c) Relations analogiques

- Termes associés (TA)

On trouvera un modèle d'entrée de thésaurus terminologique à la figure 5 .

LETTRE, nf

DF Document de correspondance externe, à caractère personnel, écrit à la main ou imprimé, généralement expédié sous enveloppe (parfois à découvert) à un ou plusieurs destinataires (institutions ou individus, identifiés ou non) et soumis à un protocole de présentation strict dit protocole épistolaire. Elle se compose d'éléments essentiels et accessoires regroupés en trois sections: les préliminaires, le corps et les compléments.

TR letter, letter mail, letter post item, lettermail, missive

TE dépêche, envoi de la poste aux lettres, missive, pli, poste-lettre, présente

TG DOCUMENT (DE) CORRESPONDANCE

TS LETTRE (D') AFFAIRES, LETTRE PERSONNELLE

TC BORDEREAU, CARTE, NOTE, TÉLÉGRAMME

TA POSTE-LETTRE SPÉCIALE

Figure 5: Modèle d'une entrée de thésaurus terminologique 


\section{CONCLUSION}

Rousseau (1983: 43-44) doute qu'il soit possible d'établir un modèle unique, une méthode universelle de la définition terminologique puisque «la conceptualisation varie en termes de niveau, de point de vue, de perception» : structure, propriétés, usages, composantes et que «la définition ne peut être conçue de la même manière selon la nature des notions et selon la nature des domaines». Il s'ensuit alors que «la structuration des notions, l'analyse notionnelle et l'élaboration des définitions sont alors forcément différentes».

Nous croyons, quant à nous, qu'il importe, avant toute chose, de préciser la nature du répertoire qui doive servir à des fins terminologiques et d'en décrire minutieusement les composantes. Ce répertoire pourrait être le thésaurus terminologique composé d'une liste systématique hiérarchisée (plan de classement) et d'une liste alphabétique structurée dont nous avons donné un modèle d'entrée. Pour vérifier la cohérence d'une telle démarche, il faut, d'abord, l'appliquer intégralement à l'intérieur d'un même domaine, puis l'étendre à plusieurs autres domaines. C'est ce à quoi nous avons travaillé (Larivière 1996).

Quoi qu'il en soit, on a beaucoup parlé de l'existence de la définition terminologique, de sa composition et de sa finalité. On y a, cependant, trouvé peu d'applications et on en a restreint la portée. En effet, on a toujours fait servir la terminologie, et partant la définition terminologique, à des fins de traduction ou d'aménagement linguistique (Rousseau 1993: 39). Il serait intéressant de savoir à quelles autres fins elle pourrait servir: procédé pédagogique d'acquisition du savoir ou technique d'expression en rédaction professionnelle. Le sachant, on pourrait ensuite évaluer les besoins réels de diverses clientèles en matière de définition pour ensuite les satisfaire.

On pourrait, par exemple, à l'instar de Sager (1990: 45-46) mais différemment, envisager trois niveaux de définitions terminologiques qui correspondraient à des besoins distincts de diverses clientèles : 1) une définition conceptuelle, minimale, qui ne se limiterait qu'à la compréhension de la notion; 2) une définition relationnelle qui inclurait, en plus, les termes génériques, spécifiques et coordonnés reliés à la notion; 3) une définition fonctionnelle dont le développement serait suffisant pour pouvoir utiliser la notion à des fins pratiques, sorte de définition élargie de type encyclopédique.

Notes

1. D'ailleurs, cette hétérogénéité dans l'emploi des définitions dans les divers répertoires a déjà été notée par Dubois (1979: 47) qui voit dans la distinction entre «dictionnaires de mots» et «dictionnaires de choses» une typologie commode au plan lexicographique mais peu conforme à la pratique lexicographique réelle. Par ailleurs, Rey (1979: 41) constate que bien des définitions de dictionnaires linguistiques et d'encyclopédies ne sont que des descriptions. Quant à de Bessé (1990: 253), il considère les définitions du Grand dictionnaire encyclopédique Larousse comme des «définitions terminographiques» qui sont «souvent précédées de définitions purement lexicographiques [et] suivies de développements nettement identifiés comme encyclopédiques».

2. Il est avantageux, en général, que ces vocabulaires fondamentaux [à définitions] soient arrangés «systématiquement», i.e. suivant une classification des notions... La raison en est que toutes les notions d'un vocabulaire fondamental doivent être définies, ce qui signifie qu'elles doivent être délimitées par rapport aux notions liées. (ISO $1968: 9$ )

3. Par ailleurs, selon Rousseau (1983: 40-41), il arrive qu'il soit nécessaire d'ajouter aux traits pertinents, dans une définition, des traits «superfétatoires» à des seules fins de compréhension pour qu'un public cible qui ne possède pas les connaissances nécessaires à la compréhension d'un message "puisse lui-même distinguer, délimiter, classer les notions et, finalement, se représenter mentalement les objets définis».

\section{RÉFÉRENCES}

BESSÉ, Bruno de (1990) : «La définition terminologique», Chaurand et Mazière (dir.), La définition: Actes du Colloque La définition, Centre d'études du lexique, Université Paris-Nord, 18-19 novembre 1988, coll. «Langue et langage», Paris, Larousse, pp. 252-261. 
CHAURAND, Jacques et Francine MAZIÈRE (dir.) (1990) : La définition: Actes du Colloque La définition, Centre d'études du lexique, Université Paris-Nord, 18-19 novembre 1988, coll, «Langue et langage», Paris, Larousse.

DUBOIS, Claude (1979) : «La spécificité de la définition en terminologie (par comparaison avec la définition en lexicographie)», Rousseau (dir.), Actes du Sixième Colloque international de terminologie, Office de la langue française, Pointe-au-Pic (Québec). 2-7 octobre 1977, Québec, Éditeur officiel du Québec, pp. 44-59.

DUQUET-PICARD, Diane (dir.) (1983) : Problèmes de la définition et de la synonymie en terminologie : Actes du Colloque international de terminologie, Université Laval, Québec, 23-27 mai 1982, organisé par GIRSTERM, OLF, DGTD avec la collaboration d' Infoterm, Québec, GIRSTERM.

GLOBENSKY, Robert (1980) : Vocabulaire de la commercialisation (anglais-français), Québec, Office de la langue française.

Grand dictionnaire encyclopédique Larousse (1982) : volume 3, Paris, Librairie Larousse.

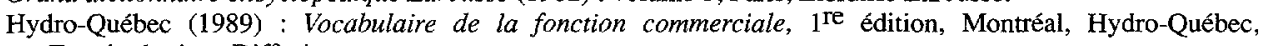
Terminologie et Diffusion.

ISO (1968) : Principes de dénomination, Recommandation ISO/R 704, 1 re édition, Genève, Organisation internationale de normalisation.

LARIVIËRE, Louise (1989) : «Vers un produit unifié en terminologie et en documentation : le thésaurus terminologique», Meta, 34 (3), pp. 457-467.

LARIVIERE, Louise (1996): Conception et réalisation d'un nouveau répertoire terminologique et documentaire unifié : le terminaire ou thésaurus TERMDOC des documents professionnels de correspondance, thèse de doctorat, 2 volumes, Université de Montréal, N.P.

LEHMANN, Alise (1990): «De définition à définition - L'interprétation dans le dictionnaire par le jeu des renvois», Chaurand et Mazière (dir.), La définition: Actes du Colloque La définition, Centre d'études du lexique, Université Paris-Nord, 18-19 novembre 1988, coll. «Langue et langage», Paris, Larousse, pp. 208-224.

McNAUGHT, John (1982) : «The Role of Terminological Relationships in Encoding and Accessing Systematic Multilingual Specialized Computer Dictionaries», Multilingua, 1 (1), pp. 53-54.

Petit Larousse illustré (1991) : Paris, Librairie Larousse.

REY, Alain (1979) : La terminologie: noms et notions, coll. «Que sais-je ?» $\mathrm{n}^{\circ} 1780$, Paris, Presses Universitaires de France.

Robert électronique (Le) (1989) : [Ensemble d'outils d'aide à la rédaction de textes français sur disque optique compact élaboré à partir du Grand Robert de la langue française de Paul Robert dans sa nouvelle édition dirigée par Alain Rey], Paris, Les dictionnaires Le Robert.

ROUSSEAU, Louis-Jean (1983) : «La définition terminologique», Duquet-Picard (dir), Problèmes de la définition et de la synonymie en terminologie: Actes du Colloque international de terminologie, Université Laval, Québec, 23-27 mai 1982, organisé par GIRSTERM, OLF, DGTD avec la collaboration d'Infoterm, Québec, GIRSTERM, pp. 47-51.

ROUSSEAU, Louis-Jean (1993) : «Terminology and Languages in Contact in Québec», Sonneveld et Loening (Eds), Terminology: Applications in Interdisciplinary Communication, Amsterdam/Philadelphia, John Benjamins Publishing Company, pp. 35-42.

SAGER, Juan Carlos (1982) : «Terminological Thesaurus: a More Appropriate Designation or a Deprecated Synonym?», Social Sciences Information Studies, 2, pp. 211-214.

SAGER, Juan Carlos (1990): A Practical Course in Terminology Processing: with a Bibliography by Blaise Nkwenti-Azeh, Amsterdam/Philadelphie, John Benjamins Publishing Company.

SONNEVELD, Helmi B. et Kurt L. LOENING (Eds) (1993) : Terminology: Applications in Interdisciplinary Communication, Amsterdam/Philadelphia, John Benjamins Publishing Company.

VILLERS, Marie-Éva de (1981) : Vocabulaire de la gestion de la production: anglais-français, Cahiers de l'Office de la langue française; Terminologie de la gestion, Québec, Office de la langue française.

\section{ANNEXE I}

VOCABULAIRE SPÉCIALISÉ (RÉPERTOIRE TERMINOLOGIQUE) :

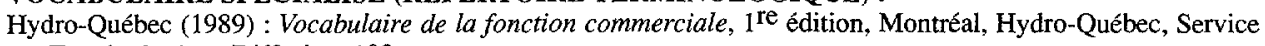
Terminologie et Diffusion, $103 \mathrm{p}$.

1) PRÉSENTATION DE LENTRÉE

contrat

Convention reconnaissant l'existence de droits et faisant naître des obligations entre les parties qui y souscrivent

VA abonnement

2) COMPOSITION DE L'ENTRÉE

unité

aucune donnée grammaticale

définition conceptuelle : incluant + traits sémantiques

renvoi Voir aussi 


\section{ANNEXE II \\ PETIT DICTIONNAIRE DE LANGUE: \\ Petit Larousse illustré (1991) : Paris, Librairie Larousse. 1) PRÉSENTATION DE L'ENTRÉE CONTRAT n.m.}

1. Convention juridique par laquelle une ou plusieurs personnes s'engagent envers d'autres personnes à faire ou à ne pas faire quelque chose. Contrat bilatéral ou synallagmatique : contrat en vertu duquel les contractants s'engagent réciproquement, les uns envers les autres (par opposition à contrat unilatéral, où seule une partie s'engage envers l'autre). - Contrat de mariage: contrat qui précise le régime des biens des époux pendant le mariage. - Contrat de travail : convention par laquelle un salarié met son activité au service d'un employeur en echange d'un salaire. - Contrat administratif, celui qui est conclu par une administration publique pour assurer un service public (marché de travaux publics, concession de service public). $>$ Remplir, réaliser son contrat: s'acquitter des obligations que l'on avait contractées, faire ce que l'on avait promis.

2. Document officiel qui constate cette convention. Rédiger, signer un contrat.

3. Contrat social: convention expresse ou tacite qui, selon J.-J. Rousseau, est conclu entre chaque individu et la communauté.

4. Au bridge, à la manille, au tarot, enchère la plus élevée déterminant le nombre de levées à réaliser.

2) COMPOSITION DE L'ENTRÉE

unité

indication grammaticale (genre, nombre)

sens de chacune des acceptions:

1 et 2: les sens du signe polysémique

3: le sens d'un terme complexe

4: le sens dans un domaine particulier

définition

1,3,4: définition conceptuelle : incluant + traits sémantiques

$2: \quad$ définition lexicale de type explicatif

emplois particuliers avec définitions conceptuelles

collocations dont le sens est expliqué par une périphrase

\section{ANNEXE III}

GRAND DICTIONNAIRE DE LANGUE:

Robert électronique (Le) (1989) : [Ensemble d'outils d'aide à la rédaction de textes français sur disque optique compact élaboré à partir du Grand Robert de la langue française de Paul Robert dans sa nouvelle édition dirigée par Alain Rey], Paris, Les dictionnaires Le Robert.

\section{1) PRESENTATION DE L'ENTRÉE}

CONTRAT [kõtRa] n. m.

1. Dr. et cour. «Convention par laquelle une ou plusieurs personnes s'obligent, envers une ou plusieurs autres, à donner, à faire ou à ne pas faire quelque chose» (Code civil, art. 1101). => Convention, pacte Contrat synallagmatique, ou bilatéral, dans lequel les contractants s'obligent réciproquement les uns envers les autres (Code civil, art. 1102). $\Rightarrow$ Echange, louage (bail, cheptel, ferme), société, vente... Contrat unilatéral, dans lequel une ou plusieurs personnes sont obligées envers une ou plusieurs autres sans aucun engagement de la part de ces dernières (Code civil, art. 1103). $\Rightarrow$ Cautionnement, dépôt, mandat, prêt, promesse. Contrat commutatif (Code civil, art. 1104). - Contrat de bienfaisance* (Code civil, art. 1105) ou à titre gratuit. $\Rightarrow$ Donation (aussi Dépôt, mandat). Contrat à titre onéreux, qui assujettit chacune des parties a donner ou à faire quelque chose (Code civil, art. 1106). $\Rightarrow$ Aléatoire, commutatif. Contrat nommé, prévu par la loi sous une dénomination propre. Contrat innommé, sans dénomination particulière et régi par les principes généraux des conventions. Contrat consensuel, produit par le seul consentement des parties. Contrat réel, produit par la livraison effective de l'objet du contrat. Contrat solennel, valable seulement quand il est revêtu des formes légales prescrites. - Contrat d'antichrèse, contrat de gage. $\Rightarrow$ Nantissement. Contrats spéciaux; contrat pignoratif, contrat mohatra. Contrat forfaitaire. $\Rightarrow$ Forfait. Contrat réglant une contestation par des concessions réciproques. $\Rightarrow$ Compromis, transaction. Contrats et quasi-contrats. Contrat judiciaire: accord de deux parties devant le juge. - Contrat d'assurance*. - Contrat de mariage, passé devant notaire et qui fixe le régime des biens des époux pendant le mariage. $\Rightarrow$ Communauté, dotal (régime dotal), séparation (de biens). Mutisme, cit. 1. - Loc. Donner des coups de canif* (cit.) dans le contrat. - Contrat de travail, se rapportant au louage de services et au louage d'industrie. - Contrat de transport, par lequel un transporteur, moyennant rémunération, fait parcourir une distance donnée à une personne, à une chose. - (1937). Contrat collectif, passé avec un groupe de personnes $\Leftrightarrow$ Concordat, convention). Contrat d'entreprise. - Contrat administratif, conclu par l'Administration en 
vue d'assurer le fonctionnement d'un service public. $\Rightarrow$ Concession, marché (de travaux publics). Contrat d'apprentissage. Contrat de vente. - Accepter (cit. 2.1) un contrat.

[1] [2] [2.1]

Les parties d'un contrat; parties au contrat: ceux qui s'engagent. $\Rightarrow$ Contractant. Capacité, consentement des parties au contrat. Validité d'un contrat. - Vices des contrats. $\Rightarrow$ Dol, erreur, violence; lésion. Objet et cause (but) licites du contrat $\Leftrightarrow$ Cause, cit. 42 et supra). Clauses d'un contrat. $\Rightarrow$ Clause (cit. 3 ), condition, disposition, stipulation. Stipulé par contrat. $\Rightarrow$ Contractuel. Passer un contrat. $\Rightarrow$ Contracter; passation Approuver, ratifier, valider un contrat. Contre-lettre modifiant un contrat. Exécuter un contrat $(\Rightarrow$ Obligation). Contrat léonin*. Rescision d'un contrat pour lésion. Révision du contrat pour imprévision. Inexécution d'un contrat. $\Rightarrow$ Contravention, dédit, dérogation, inobservation, résiliation, résolution, rupture; commissoire (clause), résolutoire, rescindable. Nullité d'un contrat. Annuler un contrat. $\Rightarrow$ Casser, dénoncer, résilier, révoquer. Expiration, suspension d'un contrat. Propagation d'un contrat.

2. Acte qui enregistre cette convention. $\Rightarrow$ Document, instrument. Contrat authentique, sous seing privé. Rédiger un contrat en bonne et due forme. Les articles d'un contrat. Le notaire a dressé le contrat. $\Rightarrow$ Instrumenter. Signer un contrat, à un contrat. Faire enregistrer un contrat. Contrat d'assurance. $\Rightarrow$ Police Cahier des charges d'un contrat administratif. Avoir son contrat en poche. Brûler, déchirer le contrat. [3]

3. Par anal. Contrat social (1762, Rousseau) : convention entre les gouvernants et les gouvernés, ou entre les membres d'une société. $\Rightarrow>$ Pacte. [4] [5] [6]

(Avec la même valeur, mais dans d'autres syntagmes). [7]

4. Bridge* contrat, où un joueur s'engage à faire un certain nombre de levées. - Réaliser son contrat (au bridge), le nombre de levées auquel on s'était engagé.

5. Réaliser, remplir son contrat, ce qu'on avait promis, ce qu'on avait fait attendre de soi.

COMP. Quasi-contrat.

\section{2) COMPOSITION DE L'ENTRÉE:}

unité suivie des traits phonétiques (transcription) et grammaticaux (genre et nombre)

sens de chacune des acceptions:

1 et $2:$ les sens du signe polysémique

3: $\quad$ le sens d'un terme complexe

4: $\quad$ le sens dans un domaine particulier

5: le sens des collocations au moyen d'une explication définition

1, 3, 4: définition conceptuelle: incluant + traits sémantiques

1: + indication du domaine (définition du Code civil)

2: définition lexicale de type explicatif

emplois particuliers avec définitions conceptuelles

autres termes du même champ sémantique : parties, vices, clauses du contrat

renvois à des termes analogiques

renvois à des citations à l'aide de numéros

indication des termes composés (ou dérivés le cas échéant)

\section{ANNEXE IV}

\section{DICTIONNAIRE ENCYCLOPÉDIQUE:}

1) PRÉSENTATION DE L'ENTRÉE

Pour cette illustration voir le Grand dictionnaire encyclopédique Larousse (1982): Volume 3, Paris, Librairie Larousse, p. 2573, à l'entrée contrat.

2) COMPOSITION DE L'ENTRÉE

unité suivie des traits grammaticaux (genre et nombre) et de l'étymologie sens de chacune des acceptions :

\section{$1,2,3:$ les sens du signe polysémique}

4: $\quad$ le sens d'un domaine particulier (argot)

5: $\quad$ le sens des collocations au moyen d'une explication

définition

$1,3,4:$ définition conceptuelle $:$ incluant + traits sémantiques

2: définition lexicale de type explicatif

emplois particuliers par domaines expliqués au moyen de définitions conceptuelles

section encyclopédique: reprend certains domaines à l'intérieur desquels plusieurs emplois sont expliqués à

l'aide, bien souvent, de définitions implicites. 